\title{
Hypergeometric Identities Related to Roberts Reductions of Hyperelliptic Integrals
}

\author{
Santosh B. Joshi and Daniele Ritelli(i)
}

\begin{abstract}
In this article starting from some reductions of hyperelliptic integrals of genus 3 into elliptic integrals, due to Michael Roberts (A Tract on the addition of Elliptic and hyperelliptic integrals, Hodger, Foster and Co, 1871) we obtain several identities which, to the best of our knowledge, are all new. The strategy used at this purpose is to evaluate Roberts integrals, in two different ways, on one side by means of elliptic integrals, obtained from the Roberts method of reduction and, on the other side, using multivariate hypergeometric functions.
\end{abstract}

Mathematics Subject Classification. 33C65, 33E05, 33C05.

Keywords. Lauricella function, complete elliptic integral of first and second kind, Appell function, Gauss hypergeometric, Cauchy-Schlömilch transformation.

\section{Introduction}

Pherhaps the most famous reduction scheme for hyperllelliptic integrals is due to Jacobi, [7] where the following identity, being $a>b>1$ and $c=$ $-(\sqrt{a}-\sqrt{b})^{2} /(1-a)(1-b)$ is established

$$
\begin{aligned}
& \int_{0}^{1} \frac{\sqrt{a b}+z}{\sqrt{z(z-1)(z-a b)(z-a)(z-b)}} \mathrm{d} z \\
& \quad=\frac{1}{\sqrt{(1-a)(1-b)}} \int_{0}^{1} \frac{\mathrm{d} x}{\sqrt{x(1-x)(1-c x)}}
\end{aligned}
$$


Identity (1.1) stems from the second degree transformation $x=(1-a)(1-$ $b) z /(z-a)(z-b)$. Generally speaking, reductions of hyperelliptic integrals are possible when a variable transformation links an hyperelliptic integral to an elliptic integral. Implications and conseguences of this situation has been exploited, for example, in the articles [11-13], in which historically famous reductions due to the aforementioned Jacobi [7], Legendre [9, 10], Hermite [6] and Goursat [4] have been used, to obtain, several identities between hypergeometric functions with different numbers of variables.

In this article, this technique is used starting from a class of reductions, probably less known, due to the Irish mathematician Micheal Roberts (18171882). A scientific biography of Roberts is provided by [18]. In [15], section 63 , Roberts presented reductions of some genus 3 hyperelliptic integrals of the form:

$$
R_{n}=\int \frac{x^{n}}{\sqrt{x^{8}-p x^{6}+q x^{4}-p x^{2}+1}} \mathrm{~d} x=\int \frac{x^{n}}{\sqrt{P(x)}} \mathrm{d} x,
$$

with $n$ non negative even integer, dealing in particular with exponents $n=$ 0, 2, 4. Reduction is achieved using the second degree Cauchy-Schlömilch transformation:

$$
u=x+\frac{1}{x} \Longleftrightarrow x=\frac{1}{2}\left(u \pm \sqrt{u^{2}-4}\right) \Longrightarrow \mathrm{d} x=\frac{1}{2}\left(1 \pm \frac{u}{\sqrt{u^{2}-4}}\right) \mathrm{d} u .
$$

For hystorical references about Cauchy-Schlömilch transformation, it is noteworthy the recent paper [1], while in [12] the use of the transformation by Legendre, very similar to the one of Roberts, is mentioned. The double sign depends on the non monotonicity of the transformation (1.3): sign + has to be taken when $x \in[0,1]$ and - when $x \in(1, \infty)$ according to Fig. 1: Transformation (1.3) generates two integrals:

$$
R_{n}=\frac{1}{2^{n-1}} \int \frac{\left(\sqrt{u^{2}-4} \pm u\right)^{n-1}}{\sqrt{u^{2}-4}} \frac{1}{\sqrt{u^{4}-(p+4) u^{2}+q+2 p+2}} \mathrm{~d} u
$$

The first integral in (1.4) holds radical $\sqrt{u^{2}-4}$ a further transformation $v=$ $\sqrt{u^{2}-4}$, gives, restricting, for instance, to $x \in[0,1]$ provides:

$$
\begin{aligned}
& R_{0}=\frac{1}{2}\left(\int \frac{\mathrm{d} u}{\sqrt{u^{4}-(p+4) u^{2}+q+2 p+2}}+\int \frac{\mathrm{d} v}{\sqrt{v^{4}-(p-4) v^{2}+q-2 p+2}}\right) \\
& R_{2}=\frac{1}{2}\left(-\int \frac{\mathrm{d} u}{\sqrt{u^{4}-(p+4) u^{2}+q+2 p+2}}+\int \frac{\mathrm{d} v}{\sqrt{v^{4}-(p-4) v^{2}+q-2 p+2}}\right) \\
& R_{4}=\frac{1}{2}\left(\int \frac{\left(1-u^{2}\right) \mathrm{d} u}{\sqrt{u^{4}-(p+4) u^{2}+q+2 p+2}}+\int \frac{\left(1+v^{2}\right) \mathrm{d} v}{\sqrt{v^{4}-(p-4) v^{2}+q-2 p+2}}\right)
\end{aligned}
$$




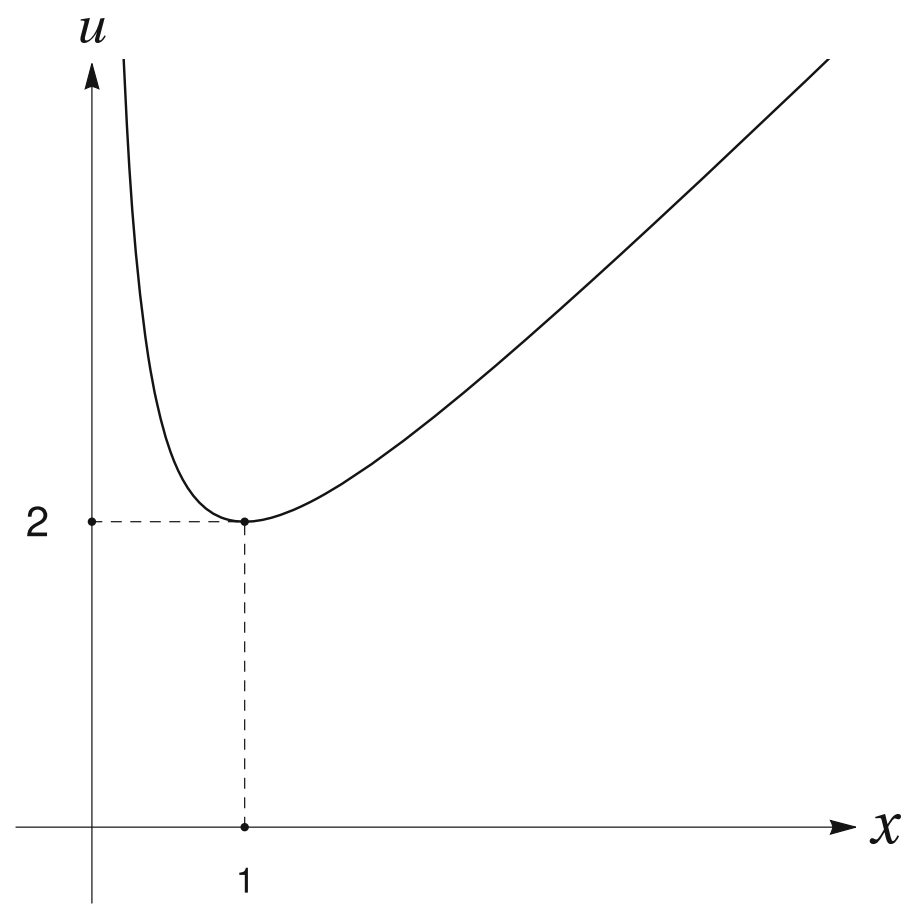

Figure 1. Cauchy-Schlömilch variable transformation

When integrating in the interval $[1, \infty)$ the sign has to be changed. In the present paper, on one side we complete Roberts's approach, producing serveral integration tables, Sect. 2, in terms of elliptic integrals, according to the different possible domains of integration, referring for simplicity only to the intervals which induce complete elliptic integrals. This contribution is of some interest in itself, given that traditional repertoires such as [2] and [5] do not report this kind of integrals. It is worth noting that Roberts's reductions are possible also for integrals with higher (even) exponents $n$ that can, as well, be reduced to elliptic integrals. In Sect. 3, as exposed in Lemmata 3.1, 3.2 and 3.3, we evaluate Roberts integrals using Lauricella functions of three or four variables establishing connections with Gauss hyperegeometric function of one variable ${ }_{2} \mathrm{~F}_{1}$ and Appell hyperegeometric function of two variables $\mathrm{F}_{1}$, Sects. 4, 5 and 6. In the arxiv preprint [14], of which this work is an extension, Roberts' results are used to obtain representations of $\pi$.

\section{The Roberts's Table of Integrals}

In order to compute Roberts integrals $R_{n}$ defined in (1.2), we, of course, need the roots of the polynomial: 


$$
P(x)=x^{8}-p x^{6}+q x^{4}-p x^{2}+1=0 .
$$

Form transformation (1.3) we obtain:

$$
x^{4}\left(x^{4}+\frac{1}{x^{4}}-p\left(x^{2}+\frac{1}{x^{2}}\right)+q\right)=x^{4}\left(\left(u^{2}-2\right)^{2}-2-p\left(u^{2}-2\right)+q\right) \text {. }
$$

Therefore roots of (2.1) are found via the auxiliary equation:

$$
u^{4}-(p+4) u^{2}+2 p+q+2=0 .
$$

Observe that if $r$ is a root of (2.1), then $-r$ and $r^{-1}$ also are roots of (2.1). Moreover, if 1 is root of (2.1) it shall be double. Hence three main situation for the roots of $P(x)=0$ can occour:

- 8 real roots

- 4 real and 2 pairs of conjugate complex roots

- 4 pairs of conjugate complex roots

Thus we can factor $P(x)$ finding two complex numbers $a, b$ such that:

$$
P(x)=\left(x^{2}-a^{2}\right)\left(x^{2}-\frac{1}{a^{2}}\right)\left(x^{2}-b^{2}\right)\left(x^{2}-\frac{1}{b^{2}}\right)
$$

This implies, using a sort of reverse engineering, that in the integrand of (1.2) the argument of the square root can be written taking $p$ and $q$ as:

$$
p=\frac{\left(a^{2}+b^{2}\right)\left(1+a^{2} b^{2}\right)}{a^{2} b^{2}}, \quad q=\frac{1+a^{4} b^{4}+\left(a^{2}+b^{2}\right)^{2}}{a^{2} b^{2}} .
$$

We will evaluate the $R_{n}$ integrals for $n=0,2,4$, in each case we provide the integration depending on the number of real roots of $P(x)=0$ expressed via (2.2) in terms of parameters $a, b$.

\subsection{Power $n=0$}

2.1.1. Subcase: $\boldsymbol{P}(\boldsymbol{x})$ has 8 Real Roots. First we introduce a specific notation to describe how (1.2) specializes when the power $n$, the integration interval, and the number of $P(x)$ 's real roots, vary. With $P(x)$ given by $(2.2)$ and $a, b \in \mathbb{R}$, with $1<a<b$, we write:

$$
\begin{aligned}
{ }_{8} R_{n}^{[0,1 / b]}(a, b) & =\int_{0}^{\frac{1}{b}} \frac{x^{n}}{\sqrt{P(x)}} \mathrm{d} x, \quad{ }_{8} R_{n}^{[1 / a, a]}(a, b)=\int_{\frac{1}{a}}^{a} \frac{x^{n}}{\sqrt{P(x)}} \mathrm{d} x, \\
{ }_{8} R_{n}^{[b, \infty)}(a, b) & =\int_{b}^{\infty} \frac{x^{n}}{\sqrt{P(x)}} \mathrm{d} x .
\end{aligned}
$$

The left index " 8 " recalls the number of real roots of (2.1); the apex concerns the integration interval; the right index marks the degree of power at numerator of each integrals, and finally the argument within round brackets defines the generators of the roots of (2.1). We will use similar notations in the following.

Using transformation (1.2) we get the following complete elliptic integrals in intervals $[0,1 / b],[1 / a, a],[b, \infty)$, which are listed in the Table 2.1 below 


\section{Table 2.1.}

$$
\begin{aligned}
& { }_{8} R_{0}^{[0,1 / b]}(a, b)=\frac{1}{2}\left(\frac{b}{1+b^{2}} K\left(\frac{b\left(1+a^{2}\right)}{a\left(1+b^{2}\right)}\right)-\frac{b}{1-b^{2}} K\left(\frac{b\left(1-a^{2}\right)}{a\left(1-b^{2}\right)}\right)\right) \\
& { }_{8} R_{0}^{[1 / a, a]}(a, b)=\frac{b}{b^{2}-1} K\left(\frac{b\left(a^{2}-1\right)}{a\left(b^{2}-1\right)}\right) \\
& { }_{8} R_{0}^{[b, \infty)}(a, b)=\frac{1}{2}\left(\frac{b}{b^{2}-1} K\left(\frac{b\left(a^{2}-1\right)}{a\left(b^{2}-1\right)}\right)-\frac{b}{b^{2}+1} K\left(\frac{b\left(a^{2}+1\right)}{a\left(b^{2}+1\right)}\right)\right)
\end{aligned}
$$

Proof. We start with (2.3). From formula (1.5) we get:

$$
\begin{aligned}
{ }_{8} R_{0}^{[0,1 / b]}(a, b)= & \frac{1}{2}\left(\int_{b+\frac{1}{b}}^{\infty} \frac{\mathrm{d} u}{\sqrt{\left[\left(a+\frac{1}{a}\right)^{2}-u^{2}\right]\left[\left(b+\frac{1}{b}\right)^{2}-u^{2}\right]}}\right. \\
& \left.+\int_{b-\frac{1}{b}}^{\infty} \frac{\mathrm{d} u}{\sqrt{\left[\left(a-\frac{1}{a}\right)^{2}-u^{2}\right]\left[\left(b-\frac{1}{b}\right)^{2}-u^{2}\right]}}\right)
\end{aligned}
$$

At right side we recognize two complete elliptic integrals of first kind, namely entries 3.151-12 page 277 of [5] or 215.00 of [2], proving (2.3). The calculation of ${ }_{8} R_{0}^{[1 / a, a]}(a, b)$ is much more laborious, since it is necessary to separate the integration interval, as anticipated in the introduction. To use (1.3) we have to split $[1 / a, a]=[1 / a, 1] \cup[1, a]$.

$$
{ }_{8} R_{0}^{[1 / a, a]}(a, b)=\int_{\frac{1}{a}}^{1} \frac{\mathrm{d} x}{\sqrt{P(x)}}+\int_{1}^{a} \frac{\mathrm{d} x}{\sqrt{P(x)}}=I_{2}^{(1)}(a, b)+I_{2}^{(2)}(a, b) .
$$

For $I_{2}^{(1)}(a, b)$ from (1.3), observing that being $x \in[0,1]$ we must choose

$$
x=1 / 2\left(u-\sqrt{u^{2}-4}\right)
$$

we get:

$$
\begin{aligned}
I_{2}^{(1)}(a, b)= & \frac{1}{2}\left(\int_{2}^{a+\frac{1}{a}} \frac{\mathrm{d} u}{\sqrt{\left[\left(a+\frac{1}{a}\right)^{2}-u^{2}\right]\left[\left(b+\frac{1}{b}\right)^{2}-u^{2}\right]}}\right. \\
& \left.+\int_{0}^{a-\frac{1}{a}} \frac{\mathrm{d} u}{\sqrt{\left[\left(a-\frac{1}{a}\right)^{2}-u^{2}\right]\left[\left(b-\frac{1}{b}\right)^{2}-u^{2}\right]}}\right)
\end{aligned}
$$


and through entries $3.152-8$ and $3.152-7$ page 276 of [5] or entries 220.00 and 219.00 of $[2]$ :

$$
\begin{aligned}
I_{2}^{(1)}(a, b)= & \frac{1}{2}\left(\frac{b}{b^{2}+1} F\left(\arcsin \frac{\left(a^{2}-1\right)\left(b^{2}+1\right)}{\left(a^{2}+1\right)\left(b^{2}-1\right)}, \frac{b\left(a^{2}+1\right)}{a\left(b^{2}+1\right)}\right)\right. \\
& \left.+\frac{b}{b^{2}-1} K\left(\frac{b\left(a^{2}-1\right)}{a\left(b^{2}-1\right)}\right)\right)
\end{aligned}
$$

For $I_{2}^{(2)}(a, b)$ the approach is similar: through the transformation $x+1 / x=$ $u$ with $x$ within $[1, a]$ with $a>1$, so that the root to be chosen is $x=$ $1 / 2\left(u+\sqrt{u^{2}-4}\right)$. As a consequence:

$$
\begin{aligned}
I_{2}^{(2)}(a, b)= & \frac{1}{2}\left(-\int_{2}^{a+\frac{1}{a}} \frac{\mathrm{d} u}{\sqrt{\left[\left(a+\frac{1}{a}\right)^{2}-u^{2}\right]\left[\left(b+\frac{1}{b}\right)^{2}-u^{2}\right]}}\right. \\
& \left.+\int_{0}^{a-\frac{1}{a}} \frac{\mathrm{d} u}{\sqrt{\left[\left(a-\frac{1}{a}\right)^{2}-u^{2}\right]\left[\left(b-\frac{1}{b}\right)^{2}-u^{2}\right]}}\right) \\
= & \frac{1}{2}\left(-\frac{b}{b^{2}+1} F\left(\arcsin \frac{\left(a^{2}-1\right)\left(b^{2}+1\right)}{\left(a^{2}+1\right)\left(b^{2}-1\right)}, \frac{b\left(a^{2}+1\right)}{a\left(b^{2}+1\right)}\right)\right. \\
& \left.+\frac{b}{b^{2}-1} K\left(\frac{b\left(a^{2}-1\right)}{a\left(b^{2}-1\right)}\right)\right)
\end{aligned}
$$

Equation (2.4) follows by adding the expressions obtained of $I_{1}^{(2)}(a, b)$ and $I_{2}^{(2)}(a, b)$.

To compute ${ }_{8} R_{0}^{[b, \infty)}(a, b)$ we see that transformation (1.3) in this situation is increasing, thus we obtain:

$$
\begin{aligned}
{ }_{8} R_{0}^{[b, \infty)}(a, b)= & \frac{1}{2}\left(\int_{b-\frac{1}{b}}^{\infty} \frac{\mathrm{d} u}{\sqrt{\left[\left(a-\frac{1}{a}\right)^{2}-u^{2}\right]\left[\left(b-\frac{1}{b}\right)^{2}-u^{2}\right]}}\right. \\
& \left.-\int_{b+\frac{1}{b}}^{\infty} \frac{\mathrm{d} u}{\sqrt{\left[\left(a+\frac{1}{a}\right)^{2}-u^{2}\right]\left[\left(b+\frac{1}{b}\right)^{2}-u^{2}\right]}}\right)
\end{aligned}
$$

Equation (2.5) follows computing the complete elliptic integrals at right-hand side through entries $3.153-11 / 12$ page 277 of [5] or 215.00 and 216.00 of [2]. 


\subsubsection{Subcase: $P(x)$ has 4 Real and 2 Pairs of Conjugate Complex Roots.} Let the polynomial (2.1) have 4 real roots and 2 pairs of conjugate complex ones which, for simplicity, we assume of modulus 1 . Then we have with $a, b \in$ $\mathbb{R}, b>1$ the following representation of $P(x)$ :

$$
P(x)=\left(x^{2}-\frac{1}{b^{2}}\right)\left(x^{2}-b^{2}\right)\left(x^{2}-e^{-2 i a}\right)\left(x^{2}-e^{2 i a}\right) .
$$

Restricting to the positive half-line, we deal with integrals on intervals $[0,1 / b]$ or $[b, \infty)$ where $P(x) \geq 0$, namely

$$
{ }_{4} R_{0}^{[0,1 / b]}(a, b)=\int_{0}^{\frac{1}{b}} \frac{\mathrm{d} x}{\sqrt{P(x)}}, \quad{ }_{4} R_{0}^{[b, \infty)}(a, b)=\int_{b}^{\infty} \frac{\mathrm{d} x}{\sqrt{P(x)}} .
$$

Transformation (1.3) applied to ${ }_{4} R_{0}^{[0,1 / b]}(a, b)$ and ${ }_{4} R_{0}^{[b, \infty)}(a, b)$ produces the second table of integrals.

\section{Table 2.2.}

$$
\begin{aligned}
& { }_{4} R_{0}^{[0,1 / b]}(a, b) \\
& \quad=\frac{1}{2}\left(\frac{b}{1+b^{2}} K\left(\frac{2 b \cos a}{1+b^{2}}\right)+\frac{b}{\sqrt{1-2 b^{2} \cos 2 a+b^{4}}} K\left(\frac{2 b \sin a}{\sqrt{1-2 b^{2} \cos 2 a+b^{4}}}\right)\right) \\
& { }_{4} R_{0}^{[b, \infty)}(a, b) \\
& \quad=\frac{1}{2}\left(\frac{b}{\sqrt{1-2 b^{2} \cos 2 a+b^{4}}} K\left(\frac{2 b \sin a}{\sqrt{1-2 b^{2} \cos 2 a+b^{4}}}\right)-\frac{b}{1+b^{2}} K\left(\frac{2 b \cos a}{1+b^{2}}\right)\right)
\end{aligned}
$$

2.1.3. Subcase: $\boldsymbol{P}(\boldsymbol{x})$ has 4 Pairs of Conjugate Complex Roots. Assume that $P(x)=0 \Longleftrightarrow|x| \leq 1$. This assumption, joined to the special $P(x)$ structure leads to:

$$
\begin{aligned}
P(x) & =\left(x^{2}-e^{2 i a}\right)\left(x^{2}-e^{-2 i a}\right)\left(x^{2}-e^{2 i b}\right)\left(x^{2}-e^{-2 i b}\right) \\
& =\left(1-2 \cos 2 a x^{2}+x^{4}\right)\left(1-2 \cos 2 b x^{2}+x^{4}\right)
\end{aligned}
$$

where $a, b$ are two real numbers. Here the table has only one entry being:

$$
{ }_{0} R_{0}^{[0, \infty)}(a, b)=\int_{0}^{\infty} \frac{\mathrm{d} x}{\sqrt{\left(1-2 \cos 2 a x^{2}+x^{4}\right)\left(1-2 \cos 2 b x^{2}+x^{4}\right)}} .
$$

Applying again tansformation (1.3), we see that (2.10) is promptly seen as a first kind complete elliptic integral, providing the unique entry of Table 2.3, where we assume $\sin ^{2} a>\sin ^{2} b$.

Table 2.3.

$$
{ }_{0} R_{0}^{[0, \infty)}(a, b)=\frac{1}{2 \sin a} K\left(\frac{\sqrt{\sin ^{2} a-\sin ^{2} b}}{\sin a}\right)
$$


Proof. Again transformation (1.3), has to be applied splitting the integration domain $[0, \infty)$ in $[0,1]$ and $[1, \infty)$ : going on with the Theorem 4.3 we find that the integral (2.10) is given by:

$$
{ }_{0} R_{0}^{[0, \infty)}(a, b)=\int_{0}^{\infty} \frac{\mathrm{d} u}{\sqrt{\left(4 \sin ^{2} a+u^{2}\right)\left(4 \sin ^{2} b+u^{2}\right)}}
$$

thus (2.11) follows from entry $3.152-1$ page 276 of [5], so that thesis follows.

\subsection{Power $n=2$}

2.2.1. Subcase: $\boldsymbol{P}(\boldsymbol{x})$ has 8 Real Roots. Assuming $1<a<b$ we use formula (1.6) applied to the following integrals:

$$
\begin{aligned}
{ }_{8} R_{2}^{[0,1 / b]}(a, b) & =\int_{0}^{\frac{1}{b}} \frac{x^{2}}{\sqrt{P(x)}} \mathrm{d} x, \quad{ }_{8} R_{2}^{[1 / a, a]}(a, b)=\int_{\frac{1}{a}}^{a} \frac{x^{2}}{\sqrt{(P(x)}} \mathrm{d} x, \\
{ }_{8} R_{2}^{[b, \infty)}(a, b) & =\int_{b}^{\infty} \frac{x^{2}}{\sqrt{P(x)}} \mathrm{d} x,
\end{aligned}
$$

being here $P(x)$ represented by $(2.2)$

\section{Table 2.4.}

$$
\begin{aligned}
{ }_{8} R_{2}^{[0,1 / b]}(a, b) & =\frac{1}{2}\left(\frac{1}{b^{2}-1} K\left(\frac{b\left(a^{2}-1\right)}{a\left(b^{2}-1\right)}\right)-\frac{1}{b^{2}+1} K\left(\frac{b\left(a^{2}+1\right)}{a\left(b^{2}+1\right)}\right)\right) \\
{ }_{8} R_{2}^{[1 / a, a]}(a, b) & =\frac{b}{b^{2}-1} K\left(\frac{b\left(a^{2}-1\right)}{a\left(b^{2}-1\right)}\right) \\
{ }_{8} R_{2}^{[b, \infty)}(a, b) & =\frac{1}{2}\left(\frac{b}{b^{2}-1} K\left(\frac{b\left(a^{2}-1\right)}{a\left(b^{2}-1\right)}\right)+\frac{b}{b^{2}+1} K\left(\frac{b\left(a^{2}+1\right)}{a\left(b^{2}+1\right)}\right)\right)
\end{aligned}
$$

Proof. The method is the same of Table 2.1, we only remark that for computing ${ }_{8} R_{2}^{[1 / a, a]}(a, b)$ it is necessary to split the interval of integration.

2.2.2. Subcase: $P(x)$ has 4 Real and 2 Pairs of Conjugate Complex Roots. The structure of $P(x)$ is again given by (2.6) so we deal, for $b>1$ and $a \in \mathbb{R}$ with integrals:

$$
{ }_{4} R_{2}^{[0,1 / b]}(a, b)=\int_{0}^{\frac{1}{b}} \frac{x^{2}}{\sqrt{P(x)}} \mathrm{d} x, \quad{ }_{4} R_{2}^{[b, \infty)}(a, b)=\int_{b}^{\infty} \frac{x^{2}}{\sqrt{P(x)}} \mathrm{d} x
$$

The relevant table of integration is 


\section{Table 2.5.}

$$
\begin{aligned}
& { }_{4} R_{2}^{[0,1 / b]}(a, b) \\
& \quad=\frac{1}{2}\left(\frac{b}{\sqrt{b^{4}-2 b^{2} \cos 2 a+1}} K\left(\frac{2 b \sin a}{\sqrt{b^{4}-2 b^{2} \cos 2 a+1}}\right)-\frac{b}{1+b^{2}} K\left(\frac{2 b \cos a}{1+b^{2}}\right)\right) \\
& { }_{4} R_{2}^{[b, \infty)}(a, b) \\
& \quad=\frac{1}{2}\left(\frac{b}{1+b^{2}} K\left(\frac{2 b \cos a}{1+b^{2}}\right)+\frac{b}{\sqrt{1-2 \cos 2 a b^{2}+b^{4}}} K\left(\frac{2 b \sin a}{\sqrt{1-2 \cos 2 a b^{2}+b^{4}}}\right)\right)
\end{aligned}
$$

2.2.3. Subcase: $\boldsymbol{P}(\boldsymbol{x})$ has 4 Pairs of Conjugate Complex Roots. Here $P(x)$ is represented by (2.9) and the unique entry of the following Table 2.6 is

$$
{ }_{0} R_{2}^{[0, \infty)}(a, b)=\int_{0}^{\infty} \frac{x^{2}}{\sqrt{\left(1-2 \cos 2 a x^{2}+x^{4}\right)\left(1-2 \cos 2 b x^{2}+x^{4}\right)}} \mathrm{d} x
$$

Aussume again $\sin ^{2} a>\sin ^{2} b$.

Table 2.6.

$$
{ }_{0} R_{2}^{[0, \infty)}(a, b)=\frac{1}{2 \sin a} K\left(\frac{\sqrt{\sin ^{2} a-\sin ^{2} b}}{\sin a}\right)
$$

\subsection{Power $n=4$}

2.3.1. Subcase: $\boldsymbol{P}(\boldsymbol{x})$ has 8 Real Roots. In this case the Cauchy-Schlömich transformation applied to Roberts's integrals, leads to a difference of two divergent integrals, this will require a passage to the limit. In addition, the exponent 4 does not allow any integration on unbounded intervals. With $P(x)$ as in (2.2) and $1<a<b$ we consider integrals:

$$
{ }_{8} R_{4}^{[0,1 / b]}(a, b):=\int_{0}^{\frac{1}{b}} \frac{x^{4}}{\sqrt{P(x)}} \mathrm{d} x, \quad{ }_{8} R_{4}^{[1 / a, a]}(a, b):=\int_{\frac{1}{a}}^{a} \frac{x^{4}}{\sqrt{P(x)}} \mathrm{d} x
$$

being, of course, ${ }_{8} R_{4}^{[b, \infty)}(a, b)=+\infty$. Assuming $1<a<b$ we write the integration table: 


\section{Table 2.7.}

$$
\begin{aligned}
{ }_{8} R_{4}^{[0,1 / b]}(a, b)= & \frac{1}{2 b}\left[\frac{\left(b^{4}-b^{2}+1\right)}{\left(1-b^{2}\right)} K\left(\frac{\left(1-a^{2}\right) b}{a\left(1-b^{2}\right)}\right)\right. \\
& +\frac{\left(b^{4}+b^{2}+1\right)}{b\left(1+b^{2}\right)} K\left(\frac{\left(a^{2}+1\right)}{a\left(b^{2}+1\right)}\right) \\
& \left.-\left(1-b^{2}\right) E\left(\frac{\left(1-a^{2}\right) b}{a\left(1-b^{2}\right)}\right)-\left(1+b^{2}\right) E\left(\frac{\left(1+a^{2}\right) b}{a\left(1+b^{2}\right)}\right)\right] \\
{ }_{8} R_{4}^{[1 / a, a]}(a, b)= & \frac{1}{b\left(b^{2}-1\right)}\left(\left(1-b^{2}+b^{4}\right) K\left(\frac{b\left(a^{2}-1\right)}{a\left(b^{2}-1\right)}\right)\right. \\
& \left.-\left(b^{2}-1\right)^{2} E\left(\frac{b\left(a^{2}-1\right)}{a\left(b^{2}-1\right)}\right)\right)
\end{aligned}
$$

Proof. To prove (2.20) we will, first, carry out the integration on $[\varepsilon, 1 / b]$ using transformation (1.3), then we take the limit for $\varepsilon \rightarrow 0^{+}$.

$$
\begin{aligned}
{ }_{8} R_{4}^{[0,1 / b]}(a, b)= & \lim _{\varepsilon \rightarrow 0^{+}} \int_{\varepsilon}^{\frac{1}{b}} \frac{x^{4}}{\sqrt{P(x)}} \mathrm{d} x \\
= & \lim _{\varepsilon \rightarrow 0^{+}}\left(\frac{1}{2} \int_{b+\frac{1}{b}}^{\varepsilon+\frac{1}{\varepsilon}} \frac{1-u^{2}}{\sqrt{\left[\left(a+\frac{1}{a}\right)^{2}-u^{2}\right]\left[\left(b+\frac{1}{b}\right)^{2}-u^{2}\right]}} \mathrm{d} u\right. \\
& \left.+\frac{1}{2} \int_{b-\frac{1}{b}}^{\varepsilon-\frac{1}{\varepsilon}} \frac{1+v^{2}}{\sqrt{\left[\left(a-\frac{1}{a}\right)^{2}-v^{2}\right]\left[\left(b-\frac{1}{b}\right)^{2}-v^{2}\right]}} \mathrm{d} v\right)
\end{aligned}
$$

In (2.22) occour two divergent integrals of the form

$$
G(u, \alpha, \beta)=\int_{\alpha}^{u} \frac{x^{2}}{\sqrt{\left(\alpha^{2}-x^{2}\right)\left(\beta^{2}-x^{2}\right)}} \mathrm{d} x
$$

where $\alpha>\beta$ and $u \rightarrow+\infty$, but in (2.22) we have to evaluate their difference. Now, by [5] entry $3.153-9$ we have:

$$
G(u, \alpha, \beta)=\alpha\left[F\left(\arcsin \frac{u}{\beta}, \frac{\beta}{\alpha}\right)-E\left(\arcsin \frac{u}{\beta}, \frac{\beta}{\alpha}\right)\right]+u \sqrt{\frac{u^{2}-\alpha^{2}}{u^{2}-\beta^{2}}}
$$

Using entries 3.151-12 page 277 of [5] and 215.00 of [2], Eq. (2.20) follows. Finally the proof of (2.21) is similar to the previous we already gave. 
2.3.2. Subcase: $P(x)$ has 4 Real and 2 Pairs of Conjugate Complex Roots. Here $P(x)$ has the form (2.6), then, we face integral:

$$
{ }_{4} R_{4}^{[0,1 / b]}(a, b)=\int_{0}^{\frac{1}{b}} \frac{x^{4}}{\sqrt{\left(x^{2}-\frac{1}{b^{2}}\right)\left(x^{2}-b^{2}\right)\left(1-2 \cos 2 a x^{2}+x^{4}\right)}} \mathrm{d} x
$$

If $b>1$ and $a \in \mathbb{R}$ we have:

Table 2.8.

$$
\begin{aligned}
{ }_{4} R_{4}^{[0,1 / b]}(a, b)= & \frac{1}{2 b}\left(\left(1+b^{2}\right) E\left(\frac{2 b \cos a}{1+b^{2}}\right)-\frac{\left(b^{4}+b^{2}+1\right)}{1+b^{2}} K\left(\frac{2 b \cos a}{1+b^{2}}\right)\right. \\
& +\frac{\left(b^{4}-b^{2}+1\right)}{\sqrt{1-2 b^{2} \cos 2 a+b^{4}}} K\left(\frac{2 b \sin a}{\sqrt{1-2 b^{2} \cos 2 a+b^{4}}}\right) \\
& \left.-\sqrt{1-2 b^{2} \cos 2 a+b^{4}} E\left(\frac{2 b \sin a}{\sqrt{1-2 b^{2} \cos 2 a+b^{4}}}\right)\right)
\end{aligned}
$$

\section{Hypergeometric Computation of Integrals $\boldsymbol{R}_{n}$}

\subsection{Hypergeometric Preliminaries}

To evaluate, using Lauricella $\mathrm{F}_{D}^{(n)}$ functions, integrals of the form:

$$
\int \frac{x^{s}}{\sqrt{\left(x^{2}-a^{2}\right)\left(x^{2}-b^{2}\right)\left(x^{2}-c^{2}\right)\left(x^{2}-d^{2}\right)}} \mathrm{d} x
$$

we need to specify several assumptions: we take $a, b, c, d$ real and such that $0<a<b<c<d$ or $a, b$ real with $0<a<b$ and $c=\bar{d}$ or $a, b, c, d$ complex with $a=\bar{b}, c=\bar{d}$. When necessary, we will impose restrictions on $s$ to ensure convergence of integral (3.1). Lemma 3.1 deals with $a, b, c, d$ all real, and further ones 3.2 and 3.3 will deal with complex roots. The hypergeometric Lauricella functions $F_{D}^{(n)}$ of $n \in \mathbb{N}^{+}$variables, introduced in the papers [16] and [8], see also the books [3] and [17] for an exhaustive introduction, is defined as:

$$
\begin{aligned}
\mathrm{F}_{D}^{(n)} & \left(\begin{array}{c}
\left.a, b_{1}, \ldots, b_{n} \mid x_{1}, \ldots, x_{n}\right) \\
c
\end{array}\right. \\
& :=\sum_{m_{1}, \ldots, m_{n} \in \mathbb{N}} \frac{(a)_{m_{1}+\cdots+m_{n}}\left(b_{1}\right)_{m_{1}} \cdots\left(b_{n}\right)_{m_{n}}}{(c)_{m_{1}+\cdots+m_{n}} m_{1} ! \cdots m_{n} !} x_{1}^{m_{1}} \cdots x_{n}^{m_{m}}
\end{aligned}
$$

where $(x)_{k}$ is the Pochhammer symbol, and under the hypergeometric series usual convergence requirements $\left|x_{1}\right|<1, \ldots,\left|x_{n}\right|<1$. If $\operatorname{Re} c>\operatorname{Re} a>0$, the relevant integral representation theorem provides:

$$
\mathrm{F}_{D}^{(n)}\left(\begin{array}{c}
a, b_{1}, \ldots, b_{n} \\
c
\end{array} \mid x_{1}, \ldots, x_{n}\right)=\frac{\Gamma(c)}{\Gamma(a) \Gamma(c-a)} \int_{0}^{1} \frac{u^{a-1}(1-u)^{c-a-1} \mathrm{~d} u}{\left(1-x_{1} u\right)^{b_{1}} \cdots\left(1-x_{n} u\right)^{b_{n}}}
$$


allowing the analytic continuation to $\mathbb{C}^{n}$ deprived of the cartesian $n$-dimensional product of the interval $(1, \infty)$ with itself. In this article we will deal with the occurrences $n=4, n=3$ and $n=2$ (Appel function).

In order to provide the hypergeometric integration, we take apart the different cases of roots of the eighth degree equation under the radical in (3.1).

\subsection{Case of 8 Real Roots}

We will restrict to $x>0$. In fact if $x \in[0, a] \cup[b, c] \cup[d, \infty)$ then $\left(x^{2}-a^{2}\right)\left(x^{2}-\right.$ $\left.b^{2}\right)\left(x^{2}-c^{2}\right)\left(x^{2}-d^{2}\right) \geq 0$. We will see the following basic definite integrals:

$$
\begin{aligned}
& J_{1}^{(s)}(a, b, c, d)=\int_{0}^{a} \frac{x^{s}}{\sqrt{\left(x^{2}-a^{2}\right)\left(x^{2}-b^{2}\right)\left(x^{2}-c^{2}\right)\left(x^{2}-d^{2}\right)}} \mathrm{d} x \\
& J_{2}^{(s)}(a, b, c, d)=\int_{b}^{c} \frac{x^{s}}{\sqrt{\left(x^{2}-a^{2}\right)\left(x^{2}-b^{2}\right)\left(x^{2}-c^{2}\right)\left(x^{2}-d^{2}\right)}} \mathrm{d} x \\
& J_{3}^{(s)}(a, b, c, d)=\int_{d}^{\infty} \frac{x^{s}}{\sqrt{\left(x^{2}-a^{2}\right)\left(x^{2}-b^{2}\right)\left(x^{2}-c^{2}\right)\left(x^{2}-d^{2}\right)}} \mathrm{d} x
\end{aligned}
$$

In Lemma 3.1, we compute (3.3), (3.4) and (3.5) in terms of Lauricella functions:

Lemma 3.1. If $0<a<b<c<d$, then:

$$
\begin{aligned}
& J_{1}^{(s)}(a, b, c, d)=\frac{a^{s} \sqrt{\pi}}{2 b c d} \frac{\Gamma\left(\frac{s+1}{2}\right)}{\Gamma\left(\frac{s+2}{2}\right)} \mathrm{F}_{D}^{(3)}\left(\begin{array}{c}
\frac{1+s}{2} ; \frac{1}{2}, \frac{1}{2}, \frac{1}{2} \\
\frac{s+2}{b^{2}}
\end{array}, \frac{a^{2}}{c^{2}}, \frac{a^{2}}{d^{2}}\right) \\
& J_{2}^{(s)}(a, b, c, d)=\frac{\pi b^{s-1}}{2 \sqrt{\left(b^{2}-a^{2}\right)\left(d^{2}-b^{2}\right)}} \\
& \mathrm{F}_{D}^{(3)}\left(\frac{1}{2} ; \frac{1-s}{2}, \frac{1}{2}, \frac{1}{2} \mid-\frac{c^{2}-b^{2}}{b^{2}},-\frac{c^{2}-b^{2}}{b^{2}-a^{2}}, \frac{c^{2}-b^{2}}{d^{2}-b^{2}}\right) \\
& J_{3}^{(s)}(a, b, c, d)=\frac{d^{s}}{2 \sqrt{\left(d^{2}-a^{2}\right)\left(d^{2}-b^{2}\right)\left(d^{2}-c^{2}\right)}} \frac{\sqrt{\pi} \Gamma\left(\frac{3-s}{2}\right)}{\Gamma\left(2-\frac{s}{2}\right)}
\end{aligned}
$$

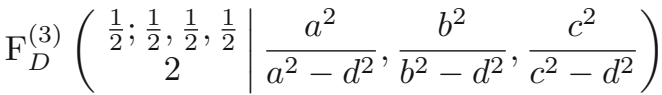

where in (3.3) and (3.6) we assume $s>-1$ and in (3.5) and (3.8) we assume $s<3$.

Proof. Through the integral representation theorem, we will compute the integrals (3.3), (3.4), (3.5). Formula (3.6) can be obtained via the change $x=a \sqrt{u}$ in (3.3) and calling (3.2). Analogously (3.7) is found putting $x=\sqrt{b^{2}+\left(c^{2}-b^{2}\right) u}$, while (3.8) follows from $x=d / \sqrt{1-u}$.

\subsection{Case of 4 Real Roots and 2 Pairs of Conjugate Complex Roots}

In Lemma 3.2 we deal with complex roots in the integrand. 
Lemma 3.2. Let $a, b \in \mathbb{R}, a<b, z \in \mathbb{C} \backslash \mathbb{R}$. Consider integrals:

$$
\begin{aligned}
& M_{1}(a, b, z)=\int_{0}^{a} \frac{x^{s}}{\sqrt{\left(x^{2}-a^{2}\right)\left(x^{2}-b^{2}\right)\left(x^{2}-z^{2}\right)\left(x^{2}-\bar{z}^{2}\right)}} \mathrm{d} x \\
& M_{2}(a, b, z)=\int_{b}^{\infty} \frac{x^{s}}{\sqrt{\left(x^{2}-a^{2}\right)\left(x^{2}-b^{2}\right)\left(x^{2}-z^{2}\right)\left(x^{2}-\bar{z}^{2}\right)}} \mathrm{d} x
\end{aligned}
$$

where in (3.9) we assume $s>-1$ and in (3.10) $s<3$. Then:

$$
\begin{aligned}
& M_{1}(a, b, z)=\frac{a^{s} \sqrt{\pi}}{2 b|z|^{2}} \frac{\Gamma\left(\frac{s+1}{2}\right)}{\Gamma\left(\frac{s+2}{2}\right)} \mathrm{F}_{D}^{(3)}\left(\begin{array}{c}
\frac{s+1}{2} ; \frac{1}{2}, \frac{1}{2}, \frac{1}{2} \\
\frac{s+2}{2}
\end{array} \frac{a^{2}}{b^{2}}, \frac{a^{2}}{z^{2}}, \frac{a^{2}}{\bar{z}^{2}}\right) \\
& M_{2}(a, b, z)=\frac{b^{s} \sqrt{\pi}}{2 \sqrt{\left(b^{2}-a^{2}\right)\left(b^{2}-z^{2}\right)\left(b^{2}-\bar{z}^{2}\right)}} \frac{\Gamma\left(\frac{3}{2}-\frac{s}{2}\right)}{\Gamma\left(2-\frac{s}{2}\right)}
\end{aligned}
$$

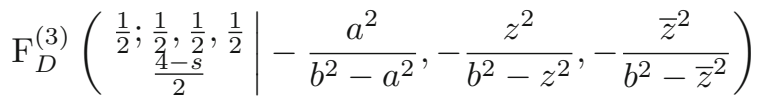

Proof. On the steps of Proof of Lemma 3.1 in the integral (3.11) the change $x=a \sqrt{u}$ is used, whilst for (3.10) the change $x=b(1-u)^{-1 / 2}$. Equations (3.11) and (3.12) follow from the integral representation theorem.

\subsection{Case of 4 Pairs of Conjugate Complex Roots}

Finally, analogous of two preceding Lemmas 3.1, 3.2 when the integrand has all its complex roots:

Lemma 3.3. Let $a, b \in \mathbb{C} \backslash \mathbb{R}$ then, if $s<3$

$$
\begin{aligned}
& L_{s}(a, b)=\int_{0}^{\infty} \frac{x^{s}}{\sqrt{\left(x^{2}-a^{2}\right)\left(x^{2}-\bar{a}^{2}\right)\left(x^{2}-b^{2}\right)\left(x^{2}-\bar{b}^{2}\right)}} \mathrm{d} x
\end{aligned}
$$

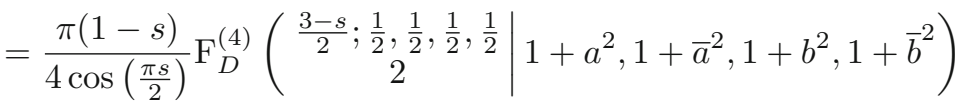

Proof. In order to apply (3.2), it will be enough a change in (3.13) putting first $x=\sqrt{v}$ and after $v=(1-u) / u$ in such a way obtaining the integral on $[0,1]$ :

$$
\frac{1}{2} \int_{0}^{1} \frac{u^{\frac{1-s}{2}}(1-u)^{-\frac{1-s}{2}}}{\sqrt{1-\left(1+a^{2}\right) u} \sqrt{1-\left(1+b^{2}\right) u} \sqrt{1-\left(1+c^{2}\right) u} \sqrt{1-\left(1+d^{2}\right) u}} \mathrm{~d} u .
$$

\section{The Hypergeometric Reductions for Power $n=0$}

We are now in position to deduce our hypergeometric reduction identities which form the core of the article. In all the entries in the integration table presented in Sect. 2 there are complete elliptic integrals of first and second kind, 
hence we will make plenty use of the well known hypergeometric representation of complete elliptic integral of first and second kind in terms of Gauss ${ }_{2} \mathrm{~F}_{1}$ :

$$
\begin{aligned}
& \frac{\pi}{2}{ }_{2} \mathrm{~F}_{1}\left(\begin{array}{c|c}
\frac{1}{2} ; \frac{1}{2} & k^{2} \\
1 &
\end{array}\right)=K(k), \\
& \frac{\pi}{2}{ }_{2} \mathrm{~F}_{1}\left(\begin{array}{c}
\frac{1}{2} ;-\frac{1}{2} \\
1
\end{array} \mid k^{2}\right)=E(k) \text {. }
\end{aligned}
$$

\subsection{Identities from Table 2.1}

Evaluating ${ }_{8} R_{0}^{[0,1 / b]}(a, b)$ via Lemma 3.1 and comparing the result with $(2.3)$ we obtain:

Theorem 4.1. If $1<a<b$ then

$$
\begin{aligned}
& \mathrm{F}_{D}^{(3)}\left(\begin{array}{c|c}
\frac{1}{2} ; \frac{1}{2}, \frac{1}{2}, \frac{1}{2} & \frac{a^{2}}{b^{2}}, \frac{1}{a^{2} b^{2}}, \frac{1}{b^{4}}
\end{array}\right) \\
& =\frac{b^{2}}{2}\left(\frac{1}{b^{2}+1}{ }_{2} \mathrm{~F}_{1}\left(\begin{array}{c}
\frac{1}{2} ; \frac{1}{2} \\
1
\end{array} \mid\left(\frac{b\left(a^{2}+1\right)}{a\left(b^{2}+1\right)}\right)^{2}\right)+\frac{1}{b^{2}-1}{ }_{2} \mathrm{~F}_{1}\left(\begin{array}{c}
\frac{1}{2} ; \frac{1}{2} \\
1
\end{array} \mid\left(\frac{b\left(a^{2}-1\right)}{a\left(b^{2}-1\right)}\right)^{2}\right)\right)
\end{aligned}
$$

Proof. We use the first integration formula of Lemma 3.1, for integral (3.3) obtaining the integration formula, which holds true for $1<a<b$ :

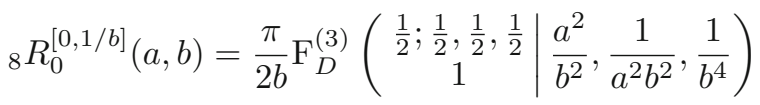

Thus (4.1) follows from (4.2) comparing the evaluation of ${ }_{8} R_{0}^{[0,1 / b]}(a, b)$ given in Table 2.1.

When one of $P(x)$ roots has the value 1 , such a root has to be double, this induces an order reduction between the three variable function $\mathrm{F}_{D}^{(3)}$ and the two variable Appell $\mathrm{F}_{1}$ :

$$
\mathrm{F}_{D}^{(3)}\left(\begin{array}{c|c}
a ; b_{1}, b_{2}, b_{3} & x, x, z \\
c &
\end{array}\right)=\mathrm{F}_{1}\left(\begin{array}{c}
a ; b_{1}+b_{2}, b_{3} \\
c
\end{array} \mid x, z\right)
$$

Thus from (4.3) we obtain a one parameter reduction relation:

Corollary 4.2. If $b>1$ then

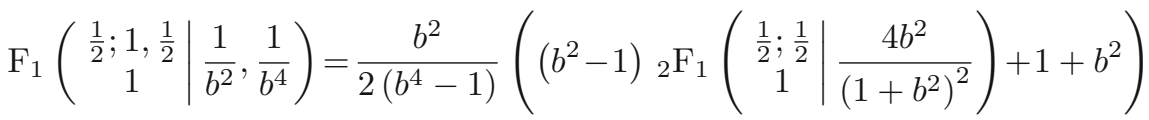

Comparing integral (2.4) with its computation obtained using Lemma 3.1, Eq. (3.7), we find out: 
Theorem 4.3. If $1<a<b$ :

$$
\begin{aligned}
& \mathrm{F}_{D}^{(3)}\left(\begin{array}{c|c}
\frac{1}{2} ; \frac{1}{2}, \frac{1}{2}, \frac{1}{2} \\
1
\end{array} \mid 1-a^{4}, \frac{\left(1-a^{4}\right) b^{2}}{b^{2}-a^{2}}, \frac{a^{4}-1}{a^{2} b^{2}-1}\right) \\
& =\frac{\sqrt{\left(b^{2}-a^{2}\right)\left(a^{2} b^{2}-1\right)}}{a^{3}\left(b^{2}-1\right)}{ }_{2} \mathrm{~F}_{1}\left(\begin{array}{c|c}
\frac{1}{2} ; \frac{1}{2} & \frac{\left(a^{2}-1\right)^{2} b^{2}}{1} \\
\left(b^{2}-1\right)^{2} a^{2}
\end{array}\right)
\end{aligned}
$$

Proof. We use Eq. (3.7) to evaluate integral ${ }_{8} R_{0}^{[1 / a, a]}(a, b)$ :

$$
\begin{aligned}
& { }_{8} R_{0}^{[1 / a, a]}(a, b) \\
& \quad=\frac{a^{3} b \pi}{2 \sqrt{\left(b^{2}-a^{2}\right)\left(a^{2} b^{2}-1\right)}} \mathrm{F}_{D}^{(3)}\left(\begin{array}{c}
\frac{1}{2} ; \frac{1}{2}, \frac{1}{2}, \frac{1}{2} \\
1
\end{array} \mid 1-a^{4}, \frac{\left(1-a^{4}\right) b^{2}}{b^{2}-a^{2}}, \frac{a^{4}-1}{a^{2} b^{2}-1}\right)
\end{aligned}
$$

Comparing (4.6) with (2.4), equation (4.5) follows.

Using Eq. (3.8) of Lemma 3.1, to evaluate integral ${ }_{8} R_{0}^{[b, \infty)}(a, b)$ comparing with (2.5) we obtain:

Theorem 4.4. Let $a<b<1$, then:

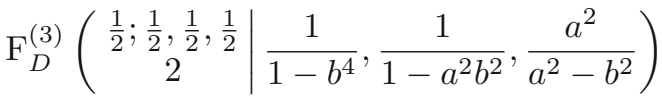

$$
\begin{aligned}
& =\frac{1}{a} \sqrt{\frac{\left(b^{2}-a^{2}\right)\left(a^{2} b^{2}-1\right)}{b^{4}-1}}\left(\left(1+b^{2}\right)_{2} \mathrm{~F}_{1}\left(\begin{array}{c|c}
\frac{1}{2} ; \frac{1}{2} & \frac{\left(a^{2}-1\right)^{2} b^{2}}{1} \\
1 & \left(b^{2}-1\right)^{2} a^{2}
\end{array}\right)\right. \\
& \left.+\left(1-b^{2}\right)_{2} \mathrm{~F}_{1}\left(\begin{array}{c|c}
\frac{1}{2} ; \frac{1}{2} & \frac{\left(a^{2}+1\right)^{2} b^{2}}{1} \\
\left(b^{2}+1\right)^{2} a^{2}
\end{array}\right)\right)
\end{aligned}
$$

Proof. By Lemma 3.1, Eq. (3.8) we have:

$$
\begin{aligned}
&{ }_{8} R_{0}^{[b, \infty)}(a, b)=\frac{\pi a b}{4} \frac{1}{\sqrt{\left(b^{4}-1\right)\left(b^{2}-a^{2}\right)\left(a^{2} b^{2}-1\right)}} \\
& \mathrm{F}_{D}^{(3)}\left(\begin{array}{c}
\frac{1}{2} ; \frac{1}{2}, \frac{1}{2}, \frac{1}{2} \\
2
\end{array} \mid \frac{1}{1-b^{4}}, \frac{1}{1-a^{2} b^{2}}, \frac{a^{2}}{a^{2}-b^{2}}\right)
\end{aligned}
$$

Formula (4.7) follows comparing (4.8) to (2.5).

Taking the limit for $a \rightarrow 1$, we obtain a one parameter reduction formula.

Corollary 4.5. If $b>1$ then

$$
\begin{aligned}
\mathrm{F}_{1} & \left(\begin{array}{c}
\frac{1}{2} ; 1, \frac{1}{2} \\
2
\end{array} \mid \frac{1}{1-b^{2}}, \frac{1}{1-b^{4}}\right) \\
& =\frac{b^{2}-1}{\sqrt{b^{4}-1}}\left(1+b^{2}+\left(1-b^{2}\right){ }_{2} \mathrm{~F}_{1}\left(\begin{array}{c}
\frac{1}{2} ; \frac{1}{2} \\
1
\end{array} \mid \frac{4 b^{2}}{\left(1+b^{2}\right)^{2}}\right)\right)
\end{aligned}
$$




\subsection{Identities from Table 2.2}

We use Lemma 3.2 to get two reduction formulas, stated in the following Theorems 4.6 and 4.7:

Theorem 4.6. If $a, b \in \mathbb{R}, b>1$ then:

$$
\begin{aligned}
& \mathrm{F}_{D}^{(3)}\left(\begin{array}{c}
\frac{1}{2} ; \frac{1}{2}, \frac{1}{2}, \frac{1}{2} \\
1
\end{array} \frac{1}{b^{4}}, \frac{e^{-2 i a}}{b^{2}}, \frac{e^{2 i a}}{b^{2}}\right)
\end{aligned}
$$

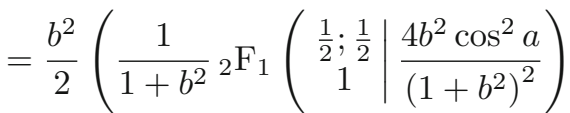

$$
\begin{aligned}
& \left.+\frac{1}{\sqrt{1-2 \cos 2 a b^{2}+b^{4}}}{ }_{2} \mathrm{~F}_{1}\left(\begin{array}{c|c}
\frac{1}{2} ; \frac{1}{2} & \frac{4 b^{2} \sin ^{2} a}{1-2 \cos 2 a b^{2}+b^{4}}
\end{array}\right)\right)
\end{aligned}
$$

Proof. Through the formula (3.11) we get the hypergeometric evaluation of ${ }_{4} R_{0}^{[0,1 / b]}(a, b)$ :

$$
{ }_{4} R_{0}^{[0,1 / b]}(a, b)=\frac{\pi}{2 b} \mathrm{~F}_{D}^{(3)}\left(\frac{1}{2} ; \frac{1}{2}, \frac{1}{2}, \frac{1}{2} \mid \frac{1}{b^{4}}, \frac{e^{-2 i a}}{b^{2}}, \frac{e^{2 i a}}{b^{2}}\right) .
$$

Equation (4.10) follows Eq. (4.11) with (2.7).

Theorem 4.7. If $a, b \in \mathbb{R}, b>1$ then

$$
\begin{aligned}
& \mathrm{F}_{D}^{(3)}\left(\begin{array}{c|c}
\frac{1}{2} ; \frac{1}{2}, \frac{1}{2}, \frac{1}{2} & \frac{1}{2} \\
\frac{1-b^{4}}{1-b^{2} e^{-2 i a}}, \frac{1}{1-b^{2} e^{2 i a}}
\end{array}\right) \\
& =\sqrt{\left(b^{4}-1\right)\left(1-2 \cos 2 a b^{2}+b^{4}\right)}
\end{aligned}
$$

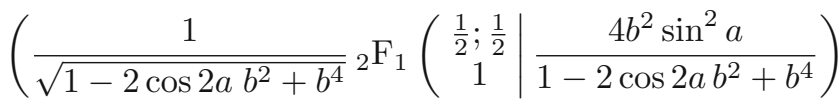

$$
\begin{aligned}
& \left.-\frac{1}{1+b^{2}}{ }_{2} \mathrm{~F}_{1}\left(\begin{array}{c|c}
\frac{1}{2} ; \frac{1}{2} & \frac{4 b^{2} \cos ^{2} a}{1} \\
\left(1+b^{2}\right)^{2}
\end{array}\right)\right)
\end{aligned}
$$

Proof. We compute integral (2.8) using Lemma 3.2 Eq. (3.10), arriving at

$$
\begin{aligned}
&{ }_{4} R_{0}^{[b, \infty)}(a, b)=\frac{b \pi}{4 \sqrt{b^{4}-1} \sqrt{1-2 \cos 2 a b^{2}+b^{4}}} \\
& \mathrm{~F}_{D}^{(3)}\left(\begin{array}{c}
\frac{1}{2} ; \frac{1}{2}, \frac{1}{2}, \frac{1}{2} \\
2
\end{array} \mid \frac{1}{1-b^{4}}, \frac{1}{1-b^{2} e^{-2 i a}}, \frac{1}{1-b^{2} e^{2 i a}}\right)
\end{aligned}
$$

Equation (4.12) follows Eqs. (4.13) and (2.8).

Remark 4.8. Notice that it is not possible, in the case of Theorems 4.6 and 4.7, take the limit $b \rightarrow 1^{+}$since both integrals (2.7), (2.8) are divergent for $b \rightarrow 1^{+}$. 


\subsection{Identities from Table 2.3}

Here we get a reduction theorem for the Lauricella $\mathrm{F}_{D}^{(4)}$ :

Theorem 4.9. Let $a, b \in \mathbb{R}$ be such that $\sin a>\sin b$. Then:

$$
\begin{aligned}
& { }_{2} \mathrm{~F}_{1}\left(\begin{array}{c|c}
\frac{1}{2} ; \frac{1}{2} & 1-\left(\frac{\sin b}{\sin a}\right)^{2} \\
1 & 1-2
\end{array}\right. \\
& =\sin a \mathrm{~F}_{D}^{(4)}\left(\frac{3}{2} ; \frac{1}{2}, \frac{1}{2}, \frac{1}{2}, \frac{1}{2} \mid 1+e^{2 i a}, 1+e^{-2 i a}, 1+e^{2 i b}, 1+e^{-2 i b}\right)
\end{aligned}
$$

Proof. Computing integral (2.10) by means of Lemma 3.3 as $L\left(e^{i a}, e^{i b}\right)$ in Eqs. (3.13), (4.14) follows, as before, by comparison.

Remark 4.10. If in (4.14) we take the limit for $a \rightarrow b$, then the Lauricella $F_{D}^{(4)}$ holds two couples of equal arguments, hence it collapses in a two-variable hypergeometric $\mathrm{F}_{1}$ Appell function according to:

$$
\mathrm{F}_{D}^{(4)}\left(\begin{array}{c}
a ; b_{1}, b_{2}, b_{3}, b_{4} \\
c
\end{array} \mid x, x, y, y\right)=\mathrm{F}_{1}\left(\begin{array}{c}
a ; b_{1}+b_{2}, b_{3}+b_{4} \\
c
\end{array} \mid x, y\right) .
$$

Thus, in the specific case $a=3 / 2, b_{1}=b_{2}=b_{3}=b_{4}=1 / 2, c=2$, since the sum of $b$ 's parameters equates $c$, we obtain the further reduction:

$$
\mathrm{F}_{1}\left(\begin{array}{c}
3 / 2 ; 1,1 \\
2
\end{array} \mid x, y\right)=\frac{2(\sqrt{1-y}-\sqrt{1-x})}{(x-y) \sqrt{(1-x)(1-y)}}
$$

which, written for $x=1+e^{2 i a}, y=1+e^{-2 i a}$ and compared with the right-hand side leads simply to the Euler identity:

$$
\frac{2}{\sqrt{-e^{-2 i a}}+\sqrt{-e^{2 i a}}}=\frac{1}{\sin a} \text {. }
$$

\section{The Hypergeometric Reductions for Power $n=2$}

\subsection{Identities from Table 2.4}

We provide more reduction formulas in Theorems 5.1, 5.3 and 5.4 starting from ${ }_{8} R_{2}^{[0,1 / b]}(a, b)$ :

Theorem 5.1. If $1<a<b$ then

$$
\begin{aligned}
\mathrm{F}_{D}^{(3)} & \left(\begin{array}{c}
\frac{3}{2} ; \frac{1}{2}, \frac{1}{2}, \frac{1}{2} \\
2
\end{array} \frac{a^{2}}{b^{2}}, \frac{1}{a^{2} b^{2}}, \frac{1}{b^{4}}\right) \\
= & \frac{b^{3}}{b^{4}-1}\left(\left(b^{2}+1\right){ }_{2} \mathrm{~F}_{1}\left(\begin{array}{c}
\frac{1}{2} ; \frac{1}{2} \\
1
\end{array} \mid\left(\frac{b\left(a^{2}-1\right)}{a\left(b^{2}-1\right)}\right)^{2}\right)\right. \\
& \left.-\left(b^{2}-1\right){ }_{2} \mathrm{~F}_{1}\left(\begin{array}{c}
\frac{1}{2} ; \frac{1}{2} \\
1
\end{array} \mid\left(\frac{b\left(a^{2}+1\right)}{a\left(b^{2}+1\right)}\right)^{2}\right)\right)
\end{aligned}
$$


Proof. Using the evaluation of ${ }_{8} R_{2}^{[0,1 / b]}(a, b)$ from the third table Eq. (5.1) thesis follows from Lemma 3.1 which provides:

$$
{ }_{8} R_{2}^{[0,1 / b]}(a, b)=\frac{\pi}{4 b^{3}} \mathrm{~F}_{D}^{(3)}\left(\frac{3}{2} ; \frac{1}{2}, \frac{1}{2}, \frac{1}{2} \mid \frac{a^{2}}{b^{2}}, \frac{1}{a^{2} b^{2}}, \frac{1}{b^{4}}\right)
$$

As in Corollary 4.2 taking the limit $a \rightarrow 1$ by (5.1) we get a one parameter reduction formula:

Corollary 5.2. If $b>1$, then:

$$
\mathrm{F}_{1}\left(\begin{array}{c}
\frac{3}{2} ; 1, \frac{1}{2} \\
2
\end{array} \mid \frac{1}{b^{2}}, \frac{1}{b^{4}}\right)=\frac{b^{4}}{b^{4}-1}\left(\left(1-b^{2}\right){ }_{2} \mathrm{~F}_{1}\left(\begin{array}{c|c}
\frac{1}{2} ; \frac{1}{2} & \frac{4 b^{2}}{1} \\
1 & \left(1+b^{2}\right)^{2}
\end{array}\right)+1+b^{2}\right)
$$

Form entry ${ }_{8} R_{2}^{[1 / a, a]}(a, b)$ of Table 2.4 we arrive at:

Theorem 5.3. If $1<a<b$, then

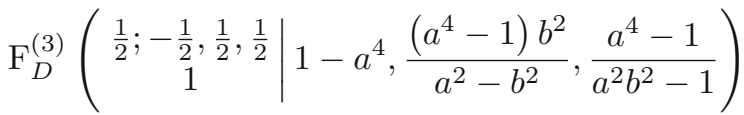

$$
\begin{aligned}
& =\frac{\sqrt{\left(b^{2}-a^{2}\right)\left(a^{2} b^{2}-1\right)}}{a\left(b^{2}-1\right)}{ }_{2} \mathrm{~F}_{1}\left(\begin{array}{c}
\frac{1}{2} ; \frac{1}{2} \\
1
\end{array} \mid\left(\frac{b\left(a^{2}-1\right)}{a\left(b^{2}-1\right)}\right)^{2}\right)
\end{aligned}
$$

Proof. Evaluating ${ }_{8} R_{2}^{[1 / a, a]}(a, b)$ using formula (3.7) of Lemma 3.1 we have:

$$
\begin{aligned}
&{ }_{8} R_{2}^{[1 / a, a]}(a, b)=\frac{\pi a b}{2 \sqrt{b^{2}-a^{2}} \sqrt{a^{2} b^{2}-1}} \\
& \mathrm{~F}_{D}^{(3)}\left(\begin{array}{c}
\frac{1}{2} ;-\frac{1}{2}, \frac{1}{2}, \frac{1}{2} \\
1
\end{array} \mid 1-a^{4}, \frac{\left(a^{4}-1\right) b^{2}}{a^{2}-b^{2}}, \frac{a^{4}-1}{a^{2} b^{2}-1}\right)
\end{aligned}
$$

Equation (5.4) follows by comparing (2.14) form Table 2.4 and (5.5) .

Theorem 5.4. If $1<a<b$ then:

$$
\begin{aligned}
\mathrm{F}_{D}^{(3)} & \left(\begin{array}{c}
\frac{1}{2} ; \frac{1}{2}, \frac{1}{2}, \frac{1}{2} \\
2
\end{array} \mid \frac{1}{1-b^{4}}, \frac{1}{1-a^{2} b^{2}}, \frac{a^{2}}{a^{2}-b^{2}}\right) \\
= & \frac{\sqrt{\left(b^{2}-a^{2}\right)\left(a^{2} b^{2}-1\right)}}{a \sqrt{b^{4}-1}}\left(\left(1+b^{2}\right){ }_{2} \mathrm{~F}_{1}\left(\begin{array}{c}
\frac{1}{2} ; \frac{1}{2} \\
1
\end{array} \mid\left(\frac{b\left(a^{2}-1\right)}{a\left(b^{2}-1\right)}\right)^{2}\right)\right. \\
& \left.+\left(1-b^{2}\right)_{2} \mathrm{~F}_{1}\left(\begin{array}{c}
\frac{1}{2} ; \frac{1}{2} \\
1
\end{array} \mid\left(\frac{b\left(a^{2}+1\right)}{a\left(b^{2}+1\right)}\right)^{2}\right)\right)
\end{aligned}
$$


Proof. Compare the evalutation of ${ }_{8} R_{2}^{[b, \infty)}(a, b)$ form Table 2.4 with formula (3.8) from Lemma 3.1, obtaining:

$$
\begin{aligned}
& { }_{8} R_{2}^{[b, \infty)}(a, b)=\frac{\pi a b^{3}}{2 \sqrt{\left(b^{4}-1\right)\left(b^{2}-a^{2}\right)\left(a^{2} b^{2}-1\right)}} \\
& \mathrm{F}_{D}^{(3)}\left(\begin{array}{c}
\frac{1}{2} ; \frac{1}{2}, \frac{1}{2}, \frac{1}{2} \\
1
\end{array} \mid \frac{1}{1-b^{4}}, \frac{1}{1-a^{2} b^{2}}, \frac{a^{2}}{a^{2}-b^{2}}\right)
\end{aligned}
$$

As usual, Eq. (5.6) follows Eqs. (2.15) and (5.7).

In this case we take, again, the limit for $a \rightarrow 1^{+}$getting by (5.6) a one parameter reduction formula similar to (4.9).

Corollary 5.5. If $b>1$ we have

$$
\begin{aligned}
b^{4} & -1-\sqrt{b^{4}-1} \mathrm{~F}_{1}\left(\begin{array}{c}
\frac{1}{2} ; 1, \frac{1}{2} \\
2
\end{array} \mid \frac{1}{1-b^{2}}, \frac{1}{1-b^{4}}\right) \\
& =\left(b^{2}-1\right)^{2}{ }_{2} \mathrm{~F}_{1}\left(\begin{array}{c}
\frac{1}{2} ; \frac{1}{2} \\
1
\end{array} \mid\left(\frac{2 b}{1+b^{2}}\right)^{2}\right)
\end{aligned}
$$

Proof. When in (5.6) we take the limit for $a \rightarrow 1^{+}$, since one of two hypergeometric becomes of argument zero it reduces to an elementary function, thus we obtain (5.8) recalling identity (4.3) already invoked in the Proof of Corollary 4.9 .

\subsection{Identities from Table 2.5}

Theorem 5.6. If $b>1$ then:

$$
\begin{aligned}
\left(1+b^{2}\right) \sqrt{b^{4}-2 b^{2} \cos 2 a+1} \mathrm{~F}_{D}^{(3)}\left(\frac{3}{2} ; \frac{1}{2}, \frac{1}{2}, \frac{1}{2} \mid \frac{1}{b^{4}}, \frac{e^{-2 i a}}{b^{2}}, \frac{e^{2 i a}}{b^{2}}\right) \\
=b^{4}\left(\left(1+b^{2}\right){ }_{2} \mathrm{~F}_{1}\left(\begin{array}{c}
\frac{1}{2} ; \frac{1}{2} \\
1
\end{array}\right)^{2}\right) \\
-\sqrt{b^{4}-2 b^{2} \cos 2 a+1}{ }_{2} \mathrm{~F}_{1}\left(\begin{array}{c}
\left.\frac{1}{2} ; \frac{1}{2} \mid\left(\frac{2 b \sin a}{1} \mid\left(\frac{2 b \cos a}{1+b^{2}}\right)^{2}\right)\right)
\end{array}\right.
\end{aligned}
$$

Proof. By Lemma 3.2 we have

$$
{ }_{4} R_{2}^{[0,1 / b]}(a, b)=\frac{\pi}{4 b^{3}} \mathrm{~F}_{D}^{(3)}\left(\frac{3}{2} ; \frac{1}{2}, \frac{1}{2}, \frac{1}{2} \mid \frac{1}{b^{4}}, \frac{e^{-2 i a}}{b^{2}}, \frac{e^{2 i a}}{b^{2}}\right)
$$

Now recalling ${ }_{4} R_{2}^{[0,1 / b]}(a, b)$ as entry of Table 2.5, Eq. (5.9) follows by comparison with (5.10). 
Theorem 5.7. If $a, b \in \mathbb{R}, b>1$ then:

$$
\begin{aligned}
& \frac{2 b^{2}}{\sqrt{b^{4}-1}} \mathrm{~F}_{D}^{(3)}\left(\begin{array}{c}
\frac{1}{2} ; \frac{1}{2}, \frac{1}{2}, \frac{1}{2} \\
1
\end{array} \mid \frac{1}{1-b^{4}}, \frac{1}{1-b^{2} e^{-2 i a}}, \frac{1}{1-b^{2} e^{2 i a}}\right) \\
& ={ }_{2} \mathrm{~F}_{1}\left(\begin{array}{c}
\frac{1}{2} ; \frac{1}{2} \\
1
\end{array} \mid\left(\frac{2 b \sin a}{\sqrt{1-2 b^{2} \cos 2 a+b^{4}}}\right)^{2}\right) \\
& +\frac{\sqrt{1-2 b^{2} \cos 2 a+b^{4}}}{1+b^{2}}{ }_{2} \mathrm{~F}_{1}\left(\begin{array}{c}
\frac{1}{2} ; \frac{1}{2} \\
1
\end{array} \mid\left(\frac{2 b \cos a}{1+b^{2}}\right)^{2}\right)
\end{aligned}
$$

Proof. Thesis follows because Lemma 3.2 establishes

$$
\begin{aligned}
& { }_{4} R_{2}^{[b, \infty)}(a, b)=\frac{b^{3} \pi}{2 \sqrt{b^{4}-1} \sqrt{1-2 \cos 2 a b^{2}+b^{4}}} \\
& \mathrm{~F}_{D}^{(3)}\left(\begin{array}{c}
\frac{1}{2} ; \frac{1}{2}, \frac{1}{2}, \frac{1}{2} \\
1
\end{array} \frac{1}{1-b^{4}}, \frac{1}{1-b^{2} e^{-2 i a}}, \frac{1}{1-b^{2} e^{2 i a}}\right)
\end{aligned}
$$

then (5.11) follows by comparison of (5.12) with entry ${ }_{4} R_{2}^{[b, \infty)}(a, b)$ of Table 2.5 .

\subsection{Identities from Table 2.6}

Regarding entry ${ }_{0} R_{2}^{[0, \infty)}(a, b)$ of Table 2.6 we take advantage of the equality

$$
{ }_{0} R_{2}^{[0, \infty)}(a, b)={ }_{0} R_{0}^{[0, \infty)}(a, b) .
$$

Integral ${ }_{0} R_{0}^{[0, \infty)}(a, b)$ at the right side of (5.13) has been computed in Theorem 4.9, formula (2.11). Hence we have:

Theorem 5.8. Let $a, b \in \mathbb{R}$ be such that $\sin a>\sin b$. Then:

$$
\begin{aligned}
& { }_{2} \mathrm{~F}_{1}\left(\begin{array}{c|c}
\frac{1}{2} ; \frac{1}{2} & 1-\left(\frac{\sin b}{\sin a}\right)^{2} \\
1 &
\end{array}\right) \\
& =4 \sin a \mathrm{~F}_{D}^{(4)}\left(\frac{1}{2} ; \frac{1}{2}, \frac{1}{2}, \frac{1}{2}, \frac{1}{2} \mid 1+e^{2 i a}, 1+e^{-2 i a}, 1+e^{2 i b}, 1+e^{-2 i b}\right)
\end{aligned}
$$

Proof. By Lemma 3.3, taking $s=2$ we obtain

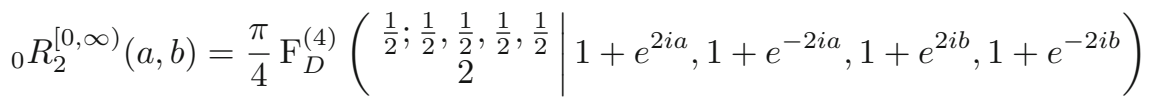

On the other side thinking ${ }_{0} R_{2}^{[0, \infty)}(a, b)$ as entry of Table 2.6, Eq. (5.14) follows comparing with (5.15).

Remark 5.9. Since the right-hand side of both identities (4.14) and (5.14) proved in Theorems 4.9 and 5.8 is the same it means that for the following 
particular choice of the arguments $\vec{v}=\left(1+e^{2 i a}, 1+e^{-2 i a}, 1+e^{2 i b}, 1+e^{-2 i b}\right)$ we have:

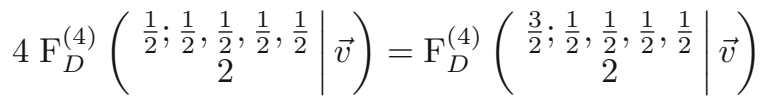

\section{The Hypergeometric Reductions for Power $n=4$}

\subsection{Identities from Table 2.7}

Using again Lemma 3.1, through the usual double evaluation, we can state:

Theorem 6.1. If $1<a<b$ then:

$$
3 \mathbb{F}=4 b^{4}(\mathbb{K}+\mathbb{E})
$$

where

$$
\begin{aligned}
& \mathbb{K}=\frac{\left(b^{4}-b^{2}+1\right)}{b^{2}-1}{ }_{2} \mathrm{~F}_{1}\left(\begin{array}{c}
\frac{1}{2} ; \frac{1}{2} \\
1
\end{array} \mid\left(\frac{\left(a^{2}-1\right) b}{a\left(b^{2}-1\right)}\right)^{2}\right) \\
& -\frac{\left(b^{4}+b^{2}+1\right)}{1+b^{2}}{ }_{2} \mathrm{~F}_{1}\left(\begin{array}{c}
\frac{1}{2} ; \frac{1}{2} \\
1
\end{array} \mid\left(\frac{\left(a^{2}+1\right) b}{a\left(1+b^{2}\right)}\right)^{2}\right)
\end{aligned}
$$

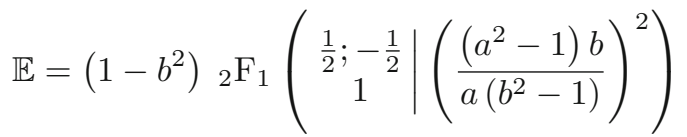

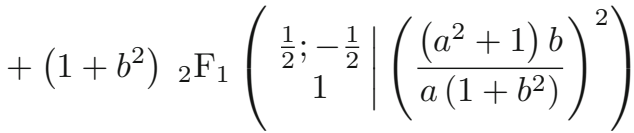

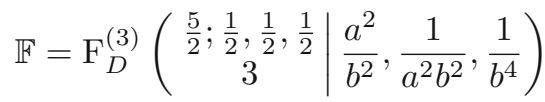

Proof. Using Lemma 3.1 to compute entry ${ }_{8} R_{4}^{[0,1 / b]}(a, b)$ of Table 2.7 we get:

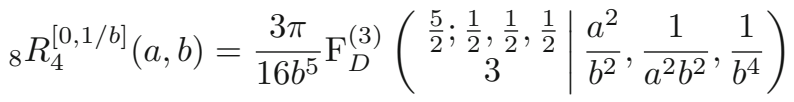

Equation (6.1) follows by comparison between (2.20) and (6.5).

Taking the limit for $a \rightarrow 1^{+}$we arrive one more time at a formula holding only one parameter with Lauricella $\mathrm{F}_{D}^{(3)}$ reducing to Appell $\mathrm{F}_{1}$. 
Corollary 6.2. If $b>1$, then

$$
\begin{aligned}
4 b^{6}+ & 3\left(1-b^{2}\right) \mathrm{F}_{1}\left(\begin{array}{c}
\frac{5}{2} ; 1, \frac{1}{2} \\
3
\end{array} \frac{1}{b^{2}}, \frac{1}{b^{4}}\right) \\
= & \frac{4 b^{4}}{1+b^{2}}\left(\left(b^{6}-1\right){ }_{2} \mathrm{~F}_{1}\left(\begin{array}{c}
\frac{1}{2} ; \frac{1}{2} \\
1
\end{array} \mid\left(\frac{2 b}{1+b^{2}}\right)^{2}\right)\right. \\
& \left.+\left(1-b^{2}\right)\left(1+b^{2}\right)^{2}{ }_{2} \mathrm{~F}_{1}\left(\begin{array}{c}
\frac{1}{2} ;-\frac{1}{2} \\
1
\end{array} \mid\left(\frac{2 b}{1+b^{2}}\right)^{2}\right)\right)
\end{aligned}
$$

By Lemma 3.1 and entry ${ }_{8} R_{4}^{[1 / a, a]}(a, b)$ of Table 2.7 we obtain:

Theorem 6.3. If $1<a<b$ then:

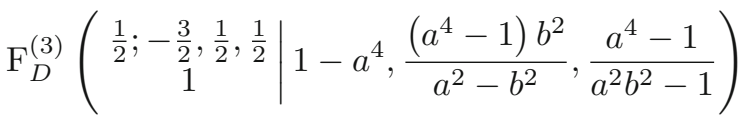

$$
\begin{aligned}
& =\frac{a \sqrt{\left(b^{2}-a^{2}\right)\left(a^{2} b^{2}-1\right)}}{b^{2}\left(b^{2}-1\right)}\left(\left(1-b^{2}+b^{4}\right){ }_{2} \mathrm{~F}_{1}\left(\begin{array}{c}
\frac{1}{2} ; \frac{1}{2} \\
1
\end{array} \mid\left(\frac{b\left(a^{2}-1\right)}{a\left(b^{2}-1\right)}\right)^{2}\right)\right. \\
& \left.-\left(b^{2}-1\right)_{2}^{2} \mathrm{~F}_{1}\left(\begin{array}{c}
\frac{1}{2} ;-\frac{1}{2} \\
1
\end{array} \mid\left(\frac{b\left(a^{2}-1\right)}{a\left(b^{2}-1\right)}\right)^{2}\right)\right)
\end{aligned}
$$

\subsection{Identities from Table 2.8}

By entry ${ }_{4} R_{4}^{[0,1 / b]}(a, b)$ [Eq. (2.24)] and by Lemma 3.2, formula (3.9), we finally state:

Theorem 6.4. If $b>1$ and $a \in \mathbb{R}$ we get:

$$
\begin{aligned}
& \frac{3}{4 b^{4}} \mathrm{~F}_{D}^{(3)}\left(\frac{5}{2} ; \frac{1}{2}, \frac{1}{2}, \frac{1}{2} \mid \frac{1}{b^{4}}, \frac{e^{-2 i a}}{b^{2}}, \frac{e^{2 i a}}{b^{2}}\right)=\left(1+b^{2}\right) \overline{\mathbb{E}}_{1}-\frac{\left(b^{4}+b^{2}+1\right)}{1+b^{2}} \overline{\mathbb{K}}_{1} \\
& +\frac{\left(b^{4}-b^{2}+1\right)}{\sqrt{1-2 b^{2} \cos 2 a+b^{4}}} \overline{\mathbb{K}}_{2}-\sqrt{1-2 b^{2} \cos 2 a+b^{4}} \overline{\mathbb{E}}_{2}
\end{aligned}
$$

where

$$
\begin{array}{ll}
\overline{\mathbb{E}}_{1}={ }_{2} \mathrm{~F}_{1}\left(\begin{array}{c}
\frac{1}{2} ;-\frac{1}{2} \\
1
\end{array} \mid A^{2}\right), & \overline{\mathbb{K}}_{1}={ }_{2} \mathrm{~F}_{1}\left(\begin{array}{c|c}
\frac{1}{2} ; \frac{1}{2} \\
1
\end{array} \mid A^{2}\right) \\
\overline{\mathbb{E}}_{2}={ }_{2} \mathrm{~F}_{1}\left(\begin{array}{c}
\frac{1}{2} ;-\frac{1}{2} \\
1
\end{array} \mid B^{2}\right), & \overline{\mathbb{K}}_{2}={ }_{2} \mathrm{~F}_{1}\left(\begin{array}{c}
\frac{1}{2} ; \frac{1}{2} \\
1
\end{array} \mid B^{2}\right)
\end{array}
$$

being

$$
A=\frac{2 b \cos a}{1+b^{2}}, \quad B=\frac{2 b \sin a}{\sqrt{1-2 b^{2} \cos 2 a+b^{4}}}
$$


Numerical control of identities (4.4) (4.9) (5.3) (5.8) (6.6)

$$
\begin{aligned}
& \ln [\circ]=\operatorname{ide} 44\left[b_{-}\right]:=\operatorname{AppetlF} 1\left[\frac{1}{2}, 1, \frac{1}{2}, 1, \frac{1}{b^{2}}, \frac{1}{b^{4}}\right]- \\
& \frac{b^{2}}{2\left(b^{4}-1\right)}\left(\left(b^{2}-1\right) \text { Hypergeometric2 F1 }\left[\frac{1}{2}, \frac{1}{2}, 1, \frac{4 b^{2}}{\left(1+b^{2}\right)^{2}}\right]+1+b^{2}\right) \\
& \operatorname{ide} 49\left[b_{-}\right]:=\operatorname{Appellt} 1\left[\frac{1}{2}, 1, \frac{1}{2}, 2, \frac{1}{1-b^{2}}, \frac{1}{1-b^{4}}\right]- \\
& \frac{b^{2}-1}{\sqrt{\left(b^{4}-1\right)}}\left(\left(1-b^{2}\right) \text { Hypergeometric2F1 }\left[\frac{1}{2}, \frac{1}{2}, 1, \frac{4 b^{2}}{\left(1+b^{2}\right)^{2}}\right]+1+b^{2}\right) \\
& \text { ide53[b_] := AppetlF1 }\left[\frac{3}{2}, 1, \frac{1}{2}, 2, \frac{1}{b^{2}}, \frac{1}{b^{4}}\right]- \\
& \frac{b^{4}}{\left(b^{4}-1\right)}\left(\left(1-b^{2}\right) \text { Hypergeometric2 } \mathrm{F} 1\left[\frac{1}{2}, \frac{1}{2}, 1, \frac{4 b^{2}}{\left(1+b^{2}\right)^{2}}\right]+1+b^{2}\right) \\
& \operatorname{ide} 58\left[b_{-}\right]:=b^{\wedge} 4-1-\sqrt{b^{\wedge} 4-1} \operatorname{AppellfF} 1\left[\frac{1}{2}, 1, \frac{1}{2}, 2, \frac{1}{1-b^{2}}, \frac{1}{1-b^{4}}\right]- \\
& \left(b^{2}-1\right)^{2}\left(\text { Hypergeometric2 } \mathrm{F} 1\left[\frac{1}{2}, \frac{1}{2}, 1, \frac{4 b^{2}}{\left(1+b^{2}\right)^{2}}\right]\right)
\end{aligned}
$$

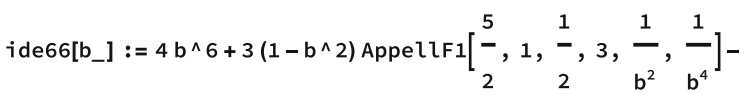

$$
\begin{aligned}
& \frac{4 b^{\wedge} 4}{1+b^{\wedge} 2}\left(\left(b^{6}-1\right) \text { Hypergeometric2F1 }\left[\frac{1}{2}, \frac{1}{2}, 1, \frac{4 b^{2}}{\left(1+b^{2}\right)^{2}}\right]+\right. \\
& \left.\left(1-b^{\wedge} 2\right)\left(1+b^{\wedge} 2\right)^{\wedge} 2 \text { Hypergeometric } 2 F 1\left[\frac{1}{2},-\frac{1}{2}, 1, \frac{4 b^{2}}{\left(1+b^{2}\right)^{2}}\right]\right) \text {; }
\end{aligned}
$$

Figure 2. Identities (4.4), (4.9), (5.3), (5.8) and (6.6) in Mathematica $_{\circledR}$

\section{Conclusions}

We obtained several identities between hypegeormetric functions which are, as far as we are concerned, all unpublished and undoubtably unknown not only to any human being but also to any computer algebra systems like Mathematica $_{\circledast}$. For example, by implementing identities (4.4), (4.9), (5.3), (5.8) and (6.6) in Mathematica ${ }_{\circledast}$ we get: Mathematica $a_{\circledR}$ is not able to reduce the above expressions. Nevertheless, as shown in Fig. 2 and Fig. 3 proceeding 


\section{$\operatorname{In}[\circ]:=\operatorname{Max}[\{\operatorname{Max}[\operatorname{Table}[\operatorname{Abs}[\mathrm{ide} 44[\mathrm{~b}]],\{b, 1.001,20, .025\}]]$, $\operatorname{Max}[\operatorname{Table}[A b s[i d e 49[b]],\{b, 1.001,20, .025\}]]$, $\operatorname{Max}[\operatorname{Table}[\operatorname{Abs}[i d e 53[b]],\{b, 1.001,20, .025\}]]$, $\operatorname{Max}[$ Table[Abs[ide58[b]] $\{b, 1.001,20, .025\}]]$, $\operatorname{Max}[\operatorname{Table}[\operatorname{Abs}[i \operatorname{de} 66[\mathrm{~b}]],\{b, 1.001,10, .025\}]]\}]$}

Out $[\circ]=2.02097 \times 10^{-7}$

Figure 3. Numerical control of (4.4), (4.9), (5.3), (5.8) and (6.6) in Mathematica $_{\circledast}$

numerically we see that the results is confirmed within the range of numerical turbulence.

\section{Acknowledgements}

The authors are indebted to Giovanni Mingari Scarpello who introduced them to Roberts' article: they hereby take the opportunity to thank him warmly. The second author is supported by Work supported by RFO 2015-2016 (Panel 13) Italian grant funding.

Funding Open access funding provided by Alma Mater Studiorum - Università di Bologna within the CRUI-CARE Agreement.

\section{Compliance with ethical standards}

Conflict of interest All the authors were involved in the preparation of the manuscript. All the authors have read and approved the final manuscript. The authors declare that they have no conflict of interest.

Open Access. This article is licensed under a Creative Commons Attribution 4.0 International License, which permits use, sharing, adaptation, distribution and reproduction in any medium or format, as long as you give appropriate credit to the original author(s) and the source, provide a link to the Creative Commons licence, and indicate if changes were made. The images or other third party material in this article are included in the article's Creative Commons licence, unless indicated otherwise in a credit line to the material. If material is not included in the article's Creative Commons licence and your intended use is not permitted by statutory regulation or exceeds the permitted use, you will need to obtain permission directly from the copyright holder. To view a copy of this licence, visit http://creativecommons. org/licenses/by/4.0/. 


\section{References}

[1] Amdeberhan, T., Glasser, M.L., Jones, M.C., Moll, V., Posey, R., Varela, D.: The Cauchy-Schlomilch transformation. Int. Trans. Special Funct. (2019). https:// doi.org/10.1080/10652469.2019.1637432

[2] Byrd, P.F., Friedman, M.D.: Handbook of Elliptic Integrals for Engineers and Scientists. Springer, Berlin (1971)

[3] Exton, H.: Multiple Hypergeometric Functions and Applications. Ellis Horwood, Chichester (1976)

[4] Goursat, E.: Sur la réduction des intégrales hyperelliptiques. Bull. de la S. M. F. 13, 143-162 (1885)

[5] Gradshteyn, I.S., Ryzhik, J.M.: Table of Integrals, Series and Products, 6th edn. Academic Press, New York (2000)

[6] Hermite, C.: Réduction d'intégrales abéliennes aux fonctions elliptiques. Ann. de la Soc. Sci. de Bruxelles 1, 1-16 (1876)

[7] Jacobi, G.: Nachschrift. J. Reine. Angew. Math. 8, 416-417 (1832)

[8] Lauricella, G.: Sulle funzioni ipergeometriche a più variabili. Rend. del Circolo Mat. di Palermo 7, 111-158 (1893)

[9] Legendre, A.M.: Traité des Fonctions Elliptiques et des Intégrales Euleriennes. Tome premier, Imprimerie de Huzard-Courcier (1825)

[10] Legendre, A.M.: Traité des Fonctions Elliptiques et des Intégrales Euleriennes. Tome second, Imprimerie de Huzard-Courcier (1826)

[11] Mingari Scarpello, G., Ritelli, D.: The hyperelliptic integrals and $\pi$. J. Number Theory 129, 3094-3108 (2009)

[12] Mingari Scarpello, G., Ritelli, D.: Legendre Hyperelliptic integrals, $\pi$ new formulae and Lauricella functions through the elliptic singular moduli. J. Number Theory 135C, 334-352 (2014)

[13] Mingari Scarpello, G., Ritelli, D.: On computing some special values of multivariate hypergeometric functions. J. Math. Anal. Appl. 420, 1693-1718 (2014)

[14] Mingari Scarpello, G., Ritelli, D.: New hypergeometric formulae to $\pi$ arising from M. Roberts hyperelliptic reductions. arXiv:1507.06681 (2015)

[15] Roberts, M.: A Tract on the Addition of Elliptic and Hyperelliptic Integrals. Hodger, Foster and Co, (1871)

[16] Saran, S.: Hypergeometric functions of three variables. Ganita 5, 77-91 (1954)

[17] Srivastava, H.M., Karlsson, P.W.: Multiple Gaussian Hypergeometric Series. Ellis Horwood, Chichester (1985)

[18] Westropp Roberts, W.: Review of the mathematical papers of the late Mr. Michael Roberts. Hermathena 5, 171-185 (1884)

Santosh B. Joshi

Department of Mathematics

Walchand College of Engineering

Sangli Maharashtra 416415

India

e-mail: santosh.joshi@walchandsangli.ac.in 
Daniele Ritelli

Departiment of Statistical Sciences

University of Bologna

via Belle Arti, 41

Bologna

Italy

e-mail: daniele.ritelli@unibo.it

Received: January 9, 2020.

Accepted: May 14, 2020.

Publisher's Note Springer Nature remains neutral with regard to jurisdictional claims in published maps and institutional affiliations. 